\section{Angioembolización selectiva en un paciente con hemorragia e inestabilidad hemodinámica provocada por la adenomectomía prostática abierta}

Bergero MA, ${ }^{1}$ Andrade-Becerra C,${ }^{1}$ Martínez $\mathrm{P},{ }^{1}$ Villamil W, ${ }^{1}$ Peralta $\mathrm{O},{ }^{2}$ GarcíaMónaco R, ${ }^{2}$ Gueglio $G,{ }^{1}$ Favre $\mathrm{G}^{1}$

\section{Resumen}

ANTECEDENTES: el sangrado abundante después de una cirugía de próstata es una complicación poco frecuente, pero grave, cuyo tratamiento requiere una nueva intervención quirúrgica. Los avances en radiología intervencionista demuestran que la angioembolización es un tratamiento efectivo en el sangrado de próstata.

CASO CLíNICO: paciente masculino de 65 años de edad, con síntomas de larga evolución de la vía urinaria inferior, en tratamiento farmacológico por hiperplasia benigna de próstata. El paciente acudió al servicio de Urgencias con retención urinaria; durante la hospitalización se le practicó adenomectomía prostática abierta. En la sala de recuperación sobrevino un evento de hemorragia abundante, con rápida disminución de la concentración de hemoglobina $(7 \mathrm{~g} / \mathrm{dL})$ e inestabilidad hemodinámica. Con la arteriografía de urgencia y embolización arterial selectiva se detuvo la hemorragia satisfactoriamente.

PALABRAS CLAVES: próstata, hemorragia, angiografía, embolización

\section{Selective angioembolization in a patient with bleeding and hemodynamic instability from open prostate adenomectomy}

Bergero MA, ${ }^{1}$ Andrade-Becerra $C,{ }^{1}$ Martínez $\mathrm{P},{ }^{1}$ Villamil W, ${ }^{1}$ Peralta $O,{ }^{2}$ GarcíaMónaco $R,{ }^{2}$ Gueglio $G,{ }^{1}$ Favre $\mathrm{G}^{1}$

\section{Abstract}

BACKGROUND: Severe bleeding after prostate surgery is an uncommon, but serious, complication that traditionally requires a new surgical intervention. Thanks to the latest advances in interventional radiology, prostatic bleeding can be successfully resolved through angioembolization.
${ }^{1}$ Departamento de Urología.

${ }^{2}$ Departamento de Diagnóstico de Imágenes, Selección de Angiografía y Terapia Endovascular. Hospital Italiano de Buenos Aires, CABA. Argentina.

Recibido: diciembre 2016

Aceptado: mayo 2017

Correspondencia

Miguel Ángel Bergero

angelbergero@hotmail.com

Este artículo debe citarse como

Bergero MA, Andrade-Becerra C, Martínez P, Villamil W, Peralta O, García-Mónaco R, Gueglio G, Favre G. Angioembolización selectiva en un paciente con hemorragia e inestabilidad hemodinámica provocada por la adenomectomía prostática abierta. Rev Mex Urol. 2017 mayo;77(3):225-229. 
CLINICAL CASE: A 65-year-old man with a lengthy history of lower urinary tract symptoms due to benign prostatic hyperplasia, and under pharmacologic treatment, arrived at the emergency room with urinary retention. During his hospitalization, the patient underwent open prostate adenomectomy. In the recovery room, he experienced severe bleeding with a rapid decrease in hemoglobin concentration $(7 \mathrm{~g} / \mathrm{dL})$ and hemodynamic instability. The patient immediately underwent emergency arteriography with selective arterial embolization that successfully resolved the bleeding.

KEY WORDS: Prostate; Bleeding; Angiography; Embolization

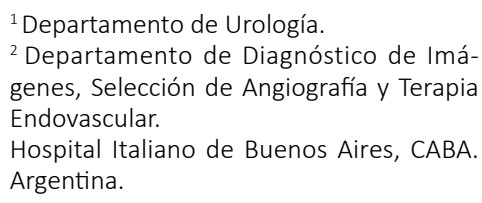

Correspondence

Miguel Ángel Bergero

angelbergero@hotmail.com

\section{ANTECEDENTES}

La angioembolización tiene un papel debidamente establecido en el tratamiento de las urgencias hemorrágicas. ${ }^{1}$ Las hemorragias urológicas (iatrogénicas, traumáticas o espontáneas, con o sin enfermedad concomitante) pueden detenerse espontáneamente, requerir un tratamiento más potente, o poner en riesgo la vida del paciente. ${ }^{1-2}$ El sangrado abundante después de una cirugía de próstata, por alguna alteración benigna, es una complicación poco frecuente, pero grave, que suele requerir reintervención abierta para detenerlo. Este procedimiento aumenta considerablemente las tasas de morbilidad y mortalidad en los pacientes. ${ }^{2}$

Los avances más recientes de la radiología intervencionista ofrecen nuevas formas de tratamiento de esta complicación. Por lo tanto, el sangrado de próstata puede detenerse con éxito mediante la embolización arterial selectiva, con baja morbilidad y mortalidad..$^{3-4}$

\section{CASO CLÍNICO}

Paciente masculino de 65 años de edad, sin comorbilidades relevantes para el padecimiento actual, con síntomas obstructivos de la vía urinaria inferior (síntomas prostáticos graves [puntuación IPSS: 27], flujometría con Q máximo de $27 \mathrm{~mL} / \mathrm{seg}$, antígeno prostático de 11 $\mathrm{ng} / \mathrm{mL}$ y biopsia prostática negativa seis meses previos a la intervención), secundarios a hiperplasia prostática, resistente al tratamiento médico (tamsulosina de $0.4 \mathrm{mg}$ /día y finasterida de 0.5 $\mathrm{mg} /$ día), por lo que se decidió no intervenirlo quirúrgicamente.

Acudió al servicio de Urgencias con retención aguda de orina. A su ingreso se le realizó examen rectal, que evidenció una próstata adenomatosa grado IV. La ecografía abdominal reportó uronefrosis bilateral leve, con $550 \mathrm{~mL}$ de orina residual y volumen prostático de $230 \mathrm{~g}$, con lóbulo medio prominente. Los estudios de laboratorio mostraron creatinina de $2.5 \mathrm{mg} / \mathrm{dL}$ y cultivo de orina negativo. Se le colocó una sonda vesical, con lo que se normalizó la concentración de creatinina $(1.2 \mathrm{mg} / \mathrm{dL})$, sin requerimiento adicional de diálisis. Durante la hospitalización se le efectuó adenomectomía prostática abierta con técnica de Millin (volumen resecado 210 g) (Figura 1). El tiempo quirúrgico fue de 85 minutos y el sangrado se estimó en $300 \mathrm{~mL}$. Sin embargo, en la sala de recuperación anestésica se observó hematuria, bloqueo del catéter vesical (que se trató con lavado), disminución 


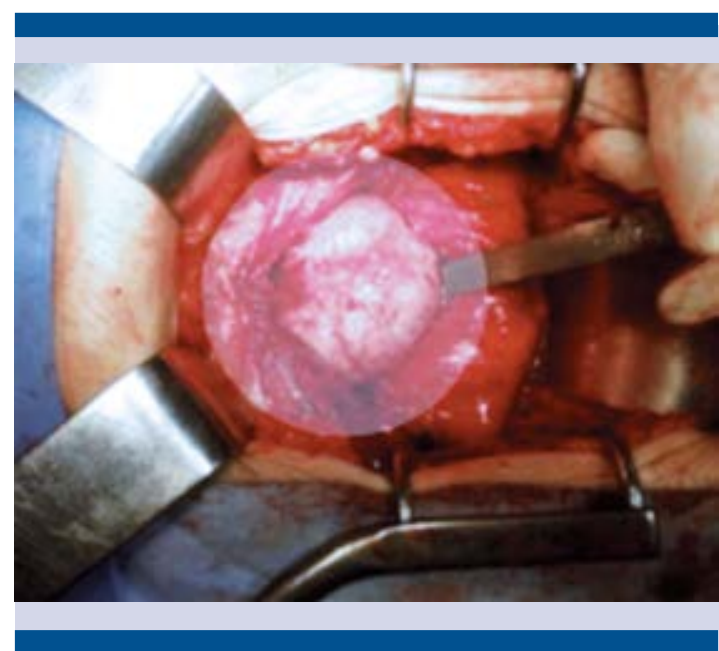

Figura 1. Adenomectomía prostática abierta: resección total del adenoma (el círculo blanco muestra un lóbulo medio prominente).

de la concentración de hemoglobina $(7 \mathrm{~g} / \mathrm{dL})$ e inestabilidad hemodinámica (taquicardia e hipotensión de 75/60 $\mathrm{mmHg}$ ) durante 10 minutos, que se estabilizó (normocardia y normotensión) con 2,000 $\mathrm{mL}$ de coloides y 3 unidades de sangre, sin requerimiento de fármacos vasopresores. Se decidió tratar el cuadro mediante un procedimiento endovascular, debido a la inestabilidad hemodinámica del paciente y la posible incapacidad de encontrar la fuente del sangrado con la intervención convencional. En la arteriografía de urgencia, por angiografía selectiva de la arteria pudenda interna derecha, se visualizó la hemorragia de la arteria capsular, detenida exitosamente con la administración de microesferas (300-500 mg). Figura 2

Después de la embolización el paciente permaneció hemodinámicamente estable (normocárdico y normotenso). No requirió trasfusiones, ni se registraron complicaciones adicionales durante la hospitalización. Al cuarto día del posoperatorio (noveno día desde su internamiento por la retención aguda de orina) se le retiraron la sonda vesical y el drenaje abdominal, y se dio de alta del hospital, con concentración de hemoglobina en parámetros normales (11 g/ dL) y diuresis espontánea.

Las citas de control médico se programaron en los días 7, 15 y 60 del posoperatorio. El estudio de histopatología reportó hiperplasia benigna de próstata. No se registraron episodios de hematuria adicionales y la concentración de hemoglobina permaneció dentro de los valores de referencia $(12.5 \mathrm{~g} / \mathrm{dL})$. En su última cita de control se identificaron síntomas prostáticos leves (puntuación I-PSS: 3), con Q máximo de 28 $\mathrm{mL} / \mathrm{seg}$ en el flujo urinario. Además, el paciente refirió buena calidad de vida, con características urinarias y función eréctil normales (puntuación IIEF-5: 21).

\section{DISCUSIÓN}

La hemorragia abundante por adenomectomía prostática abierta es excepcional, aunque potencialmente mortal y un reto terapéutico. El tratamiento habitual de esta complicación requiere una nueva intervención quirúrgica o un procedimiento endoscópico; sin embargo, estas intervenciones pueden dificultar la visualización de la hemorragia y la implementación del tratamiento oportuno. ${ }^{2}$

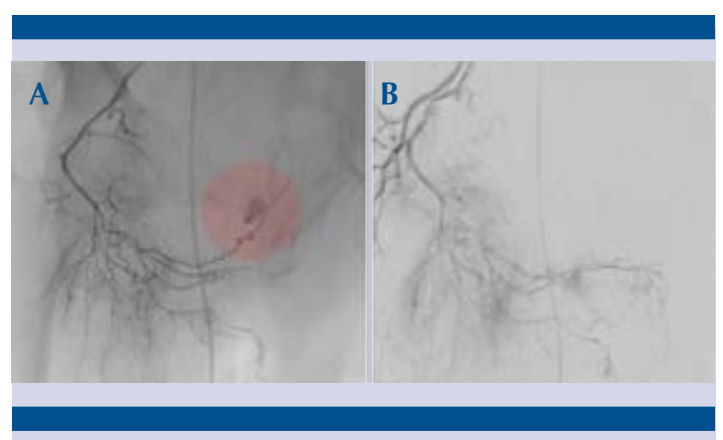

Figura 2. Angiografía: A) arteria pudenda interna derecha con sangrado proveniente de la arteria capsular (círculo rojo) y B) embolización selectiva del sangrado con administración de microesferas (300-500 mg). 
En las últimas décadas, los métodos angiográficos combinan los procedimientos diagnósticos y terapéuticos, y han demostrado confiabilidad en la detección de la hemorragia y su detención. ${ }^{1}$

En la actualidad, la angiografía es un método útil para tratar diferentes tipos de sangrado provenientes del aparato urinario. La hemorragia renal (iatrogénica, espontánea o traumática) es frecuente y la angiografía ha demostrado detenerla en más de $90 \%$ de los casos. ${ }^{1}$ A pesar de los buenos resultados reportados en las hemorragias de origen renal, la angiografía es un estudio poco efectivo en el tratamiento del sangrado vesical; esta diferencia se atribuye a que en este grupo de pacientes, algunos sufren de cistitis actínica con sangrados recurrentes.

Hoy en día existe poca evidencia que respalde la eficacia de la embolización en el tratamiento de la hemorragia provocada por la cirugía de próstata, porque este procedimiento rara vez está indicado para detener esa complicación. ${ }^{3-4}$

Algunos estudios reportan casos aislados de cohibición exitosa de la hemorragia secundaria a procedimientos prostáticos (por ejemplo, la resección transuretral de la próstata) mediante angiografía. ${ }^{4}$ Un estudio efectuado en pacientes con hemorragia del aparato urinario mostró eficacia de $90 \%$, baja morbilidad y mortalidad para detener el sangrado prostático cuando la arteria prostática es correctamente identificada mediante la angiografía. ${ }^{3}$

La exploración quirúrgica de urgencia era el método de elección para el tratamiento de la hemorragia en pacientes hemodinámicamente inestables; sin embargo, en la actualidad está demostrada la eficacia de la angiografía en el tratamiento de pacientes con hemorragia traumática aguda e inestabilidad hemodinámica. ${ }^{5} \mathrm{Tal}$ es el caso de que en muchos centros de salud, la angiografía se ha convertido en el estudio de primera línea para detener el sangrado de pacientes hemodinámicante inestables. ${ }^{5}$

En el paciente de este estudio se observó hemorragia abundante e inestabilidad hemodinámica después de una cirugía de próstata. Debido a estos hallazgos se tomó la decisión de recurrir a la angiografía, con posterior embolización, como la mejor opción terapéutica para salvar la vida del paciente. En caso de haber practicado la cirugía de urgencia, el resultado habría sido incierto; además, se trató de manera similar a una hemorragia renal. Primero se estabilizó al paciente y después se realizó la angioembolización selectiva para detener la hemorragia, con la que se obtuvieron resultados favorables.

Ante un evento de hemorragia abundante e inestabilidad hemodinámica por adenomectomía prostática abierta, la angioembolización arterial selectiva de las arterias prostáticas representa una opción de tratamiento efectiva.

\section{Fuente de financiamiento}

El trabajo no tuvo fuente de financiamiento.

\section{Conflicto de interés}

Los autores declaran no tener conflictos de interés.

\section{REFERENCIAS}

1. Sayani R, Azeemuddin M, ul Haq T, Hamid RS, Salam B. An institutional review of transarterial embolization in haemorrhagic urological emergencies. J Pak Med Assoc 2012;62(2):107-11.

2. Gaya-Sopena JM, Arce-Gil J, Gausa-Gascón L, MontlleóGonzález M, Salvador-Bayarri J, Villavicencio-Mavrich H. Transurethral treatment of massive hematuria post retropubic adenomectomy. Actas Urol Esp 2007;31(7):743-5.

3. Delgal A, Cercueil JP, Koutlidis N, Michel F, Kermarrec I, Mourey $E$, Cormier L, Krausé D, Loffroy R. Outcome of transcatheter arterial embolization for bladder and prostate hemorrhage. J Urol 2010;183:1947-53. 
4. Barbieri A, Simonazzi M, Marcato C, Larini P, Barbagallo $M$, Frattini A, Cortellini P. Massive hematuria after transurethral resection of the prostate: management by intraarterial embolization. Urol Int 2002;69(4):318-20.
5. Brewer ME Jr, Strnad BT, Daley BJ, Currier RP, Klein FA, Mobley JD, Kim ED. Percutaneous embolization for the management of grade 5 renal trauma in hemodynamically unstable patients: initial experience. J Urol 2009;181(4):1737-41.

\section{AVISO PARA LOS AUTORES}

Revista Mexicana de Urología tiene una nueva plataforma de gestión para envío de artículos: https://www.revisionporpares.com/index.php/RMUrol ahí podrá inscribirse a la base de datos administrada por el sistema Open Journal System (OJS) que ofrece las siguientes ventajas para los autores:

- Subir sus artículos directamente al sistema.

- Conocer, en cualquier momento, el estado de los artículos enviados, es decir, si ya fueron asignados a un revisor, aceptados con o sin cambios, o rechazados.

- Participar en el proceso editorial corrigiendo y modificando sus artículos hasta su aceptación final. 